\title{
Perception of reduced words: Chunking and predictability
}

David Lorenz ${ }^{1}$, David Tizón-Couto ${ }^{2}$

${ }^{1}$ English Department, Albert-Ludwigs-Universität Freiburg, Germany

${ }^{2}$ Facultade de Filoloxía e Tradución, Universidade de Vigo, Spain

https://doi.org/10.36505/ExLing-2016/07/0021/000280

\begin{abstract}
This is a first report on a word-monitoring experiment to examine how frequencybased chunking and predictability affect recognition of reduced speech. The effect of reduction on recognition of the word to was tested in English $\mathrm{V}$ to $\mathrm{V}_{\text {inf }}$ constructions of varying frequencies (e.g. have to go, prefer to stay). Our first results suggest that in types of mid-high frequency, predictability aids the recognition of a reduced item. In very high frequency sequences, however, reduction seems to encourage chunking, that is, accessing the sequence as a single unit.
\end{abstract}

Key words: chunking, reduction, frequency, speech perception

\section{Introduction}

It has long been noted that certain multi-word sequences undergo phonological reduction and contraction to a single word (e.g. want to $>$ wanna). In usage-based approaches, this is seen as a matter of coalescence, or chunking, which in turn has been linked to frequency (i.a. Bybee 2006, Ellis et al. 2009). Thus high-frequency sequences will be stored in the mind as a single unit. They have a propensity for reduction due to neuromotor routines (Bybee 2006), but the reduced forms may be more or less strongly represented in the language user's mind, on a gradient cline from on-line reduction in articulation to stored, fixed variants (Connine \& Pinnow 2006, Lorenz 2013).

Most of the evidence of chunking and the gradient status of reductions regards language production only, which raises the question how they affect speech perception. There is some evidence that full canonical forms generally serve the listener best (Tucker 2011, Pitt et al. 2011). In a word recognition experiment, Sosa \& MacFarlane (2002) show that listeners treat highly frequent sequences as chunks, leading to a delayed recognition of elements of the sequence (e.g. of in kind off). Their design did not, however, consider these sequences' propensity for reduction (e.g. "kinda") and its effect on word recognition. In a similar study Kapatsinski \& Radicke (2009) find a U-shaped frequency effect, such that word recognition is delayed in sequences of both very high and very low frequency. They suggest that frequent co-occurrence increases

ExLing 2016: Proceedings of $7^{\text {th }}$ Tutorial and Research Workshop on Experimental Linguistics, 27 June - 2 July 2016, Saint Petersburg, Russia 
the predictability of a word, hence facilitates its recognition, and that this is offset by chunking and low salience in collocations of very high frequency.

The present study builds up on this, testing the import of string frequency and reduction on speech perception. It employs constructions of the type $\mathrm{V}$ to $\mathrm{V}_{\text {inf }}$ (e.g. need to work, dare to go) to measure response times to the word to.

The crucial question is how frequency and reduction interact. In high frequency collocations, listeners may have an active knowledge of the high probability of to based on frequency, leading to a higher expectation of reduction (cf. Jurafsky et al. 2001); in this case reduction would not strongly affect recognition times. On the other hand, listeners may have a chunked item available; in that case a reduced form would lead them to access this chunked variant and considerably delay recognition of to.

\section{Experiment design}

The stimuli consist of 126 recorded sentences in American English. 42 of these contain a $\mathrm{V}$ to $\mathrm{V}_{\text {inf }}$ construction (the target items), 42 contain to in a different construction (control items), 42 do not contain to at all (distractors). Native speakers of American English were asked to respond to the presence or absence of to as accurately and quickly as possible. Response times were measured from the onset of $t$.

The $\mathrm{V}$ to $\mathrm{V}_{\text {inf }}$ sequences are of varying frequencies, as taken from the Corpus of Contemporary American English (COCA, Davies 2008-) e.g. trying to $\mathrm{V}_{\text {inf }}$ (high frequency), deign to $\mathrm{V}_{\text {inf }}$ (low frequency). Participants were assigned to one of two groups; each group heard half of the target items with a full pronunciation, the other half with a reduced to (e.g. need to as "needa"). This reduction and the frequency of the sequence serve as independent variables whose effect on response times is tested.

At the time of writing, the study is still ongoing. We present here a sketch of the results from 22 participants, which gives a first impression of the interplay of frequency and reduction.

\section{Results}

Overall, participants correctly identified to within 2000 milliseconds in $89.7 \%$ of cases $(1658 / 1848)$. When comparing conditions, however, the accuracy rate is significantly lower for reduced items than for fully articulated ones $(82.7 \%$ vs $94.4 \%)$.

There is also a clear difference between full and reduced stimuli in the response times of the correct responses. Recognition of reduced 
items is significantly delayed compared to full items. The mean response times are:

Full to: $636 \mathrm{~ms}$ - Reduced to: $786 \mathrm{~ms}$ - Control: $683 \mathrm{~ms}$

Response times to full and reduced items of different frequencies are shown in Fig.1. The four frequency bins are derived from the surface frequencies of the $\mathrm{V}$ to $\mathrm{V}_{\text {in }}$ types in COCA, ' 1 ' being the lowest frequency (up to 1.5 occurrences per 1 million words), '4' the highest (over 290 per million).

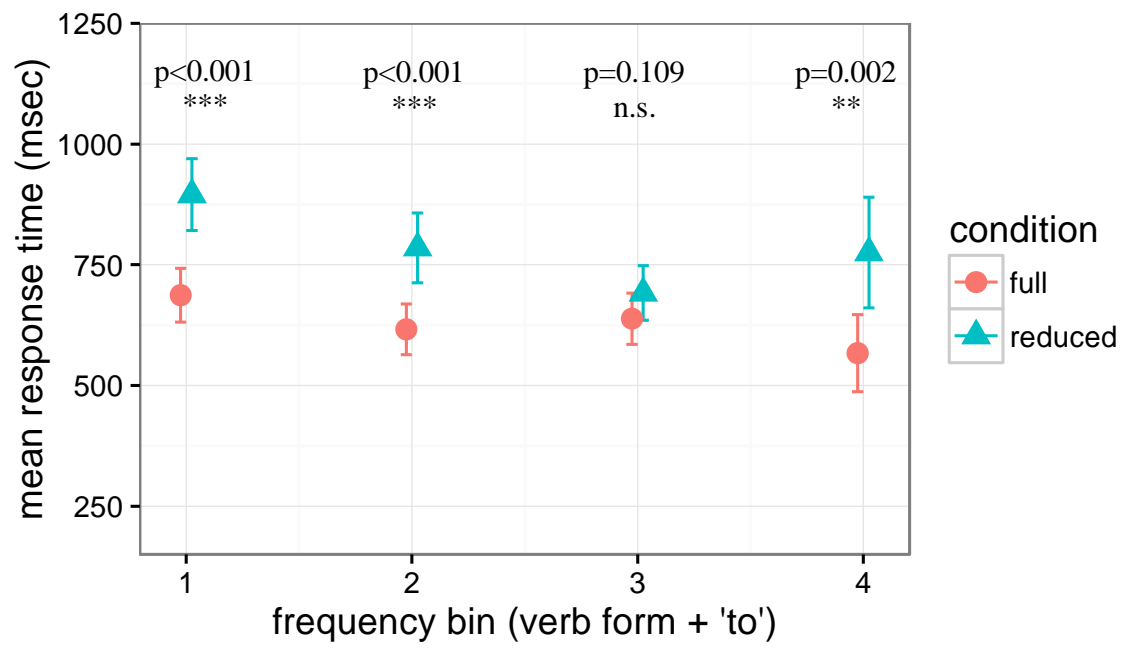

Figure 1. Response times to full and reduced to by frequency of $\mathrm{V}$ to $\mathrm{V}_{\text {inf }}$ type. The $\mathrm{p}$ values refer to Mann-Whitney U test of difference between 'full' and 'reduced' in each frequency bin.

As Fig.1 shows, there is a clear difference between response times to full and reduced items, except at mid-high frequencies (bin 3). Recognition of reduced to is slowed down at low and very high frequencies. The pattern is less clear for the fully pronounced items, where recognition appears to be less sensitive to frequency.

\section{Discussion}

In low frequency collocations (bin 1), to is least predictable from context, and reduction will be least expected; here its recognition is slowest in both full and reduced forms.

Regarding the pattern for reduced items in Fig.1, our tentative interpretation is that there is a frequency range (around or within bin 3) at which to is highly predictable and reduction can be expected; therefore, reduction does not inhibit recognition. At higher frequencies 
(bin 4), a chunking effect sets in which inhibits recognition of the element and which is reinforced by a reduced rendering. Possibly, this chunking also implies an expectation of reduction, such that a reduced input leads the listener onto a non-compositional access path (making it more difficult to retrieve the element to), whereas the non-reduced form encourages a compositional interpretation and thus does not inhibit recognition of the element.

These results need to be checked against possible other factors such as the form and length of the verb preceding to. It also remains to be seen how the frequency measure employed here - surface frequency of construction types - compares to measures of transitional probability or mutual information. In general, the findings suggest that hearers use probabilistic and frequency information to cope with reduction in the flow of speech.

\section{References}

Bybee, J. 2006. From usage to grammar: The mind's response to repetition. Language 82(4), 711-733.

Connine, C. and Pinnow, E. 2006. Phonological variation in spoken word recognition: Episodes and abstractions. The Linguistic Review 23, 235-245.

Davies, Mark. 2008-. The Corpus of Contemporary American English: 450 million words, 1990-present. Available online at http://corpus.byu.edu/coca/.

Ellis, N., Frey, E. and Jalkanen, I. 2009. The psycholinguistic reality of collocation and semantic prosody (1): Lexical access. In Römer, U. and Schulze, R. (eds.) 2009, Exploring the Lexis-Grammar Interface, 89-114. Amsterdam, John Benjamins.

Jurafsky, D., Bell, A., Gregory, M. and Raymond, W. 2001. Probabilistic relations between words: Evidence from reduction in lexical production. In Bybee, J. and Hopper, P. (eds.) 2001, Frequency and the Emergence of Linguistic Structure, 229254. Amsterdam, John Benjamins.

Kapatsinski, V. and Radicke, J. 2009. Frequency and the emergence of prefabs: Evidence from monitoring. Formulaic Language 2, 499-520.

Lorenz, D. 2013. Contractions of English Semi-Modals: The Emancipating Effect of Frequency. NIHIN Studies. Freiburg, Rombach.

Pitt, M., Dilley, L. and Tat, M. 2011. Exploring the role of exposure frequency in recognizing pronunciation variants. Journal of Phonetics 39, 304-311.

Sosa, A. and MacFarlane, J. 2002. Evidence for frequency-based constituents in the mental lexicon: collocations involving the word of. Brain and Language 83, 227-236.

Tucker, B. 2011. The effect of reduction on the processing of flaps and /g/ in isolated words. Journal of Phonetics 39, 312-318. 